\title{
Neopterin in patients with chronic kidney disease and patients with coronary artery disease
}

\author{
DOROTA FORMANOWICZ \\ Department of Clinical Biochemistry and Laboratory Medicine, Poznan University of Medical Sciences, Poznań, Poland \\ Corresponding author: doforman@ump.edu.pl
}

\begin{abstract}
Neopterin, a marker of stimulated cellular immune response, levels increase during many disorders. A number of different features of neopterin prompted us to study its serum concentration in patients with all stages of chronic kidney disease (CKD) and patients with coronary artery disease (CARD). To the best of our knowledge this is the first study that evaluates and compares the serum neopterin concentration in those diseases taken together as one study. One hundred and twenty five subjects, divided into five groups, were included in the study. The first three groups consisted of patients with different stages of CKD. CARD patients without CKD and healthy volunteers as controls were also studied. Serum neopterin concentration was measured by an enzyme-linked immunosorbent assay (ELISA, BRAHMS, Hennigsdorf/Berlin, Germany) according to the manufacturers' instructions. We have found that patients with the most advanced stage of CKD had fifteen times higher serum neopterin than patients from other studied groups. Interestingly, in the CKD1-2, CKD3-4 and CARD patients without CKD, serum neopterin concentrations were similar to those obtained from healthy volunteers. Serum urea and serum ferritin appeared to be significant independent predictors of serum neopterin concentration after adjustment for urea, CA-IMT, peripheral systolic blood pressure, ferritin, hsCRP, HD vintage, BMI and eGFR, explaining $95.89 \%$ of serum neopterin variations. In conclusion, it seems that despite the existence of many factors that may influence serum neopterin, those derived from impaired renal function and immunological disturbances coming from hemodialysis treatment are crucial. In this study, we observed that the more impaired the renal function, higher is the increase in serum neopterin concentration.
\end{abstract}

Key words: neopterin, atherosclerosis, chronic kidney disease, immunological disturbances, inflammatory process

\section{Introduction}

Neopterin (D-erythro-1', 2', 3'-trihydroxypropylpterinpteridine) is derived from guanosine- $5^{\prime}$-triphosphate (GTP) and is produced by monocytes and macrophages upon stimulation with interferon-gamma (IFN-gamma). IFN-gamma is synthesized in early innate nonspecific response of the immune system by activated T-lymphocyes and natural killer cells (Hueber et al., 1984; Murr et al., 2002) and therefore the concentration of neopterin reflects the degree of Th1-type immune activation. Thus, the amount of neopterin in human body changes under many conditions in which cellular immune system activation occurs (Fuchs et al., 1993), such as viral infections (Singh et al., 2010), inflammatory diseases, autoimmune diseases, various malignant disorders, allograft rejections (Margreiter et al., 1983), neurological and cardiovascular diseases (Fuchs et al., 1992). The cellular im- mune system is involved in the pathogenesis of all the above-mentioned diseases and neopterin concentrations are very closely linked with their progression.

Moreover, it was discovered that IFN-gamma, crucial for neopterin synthesis, also stimulates the release of reactive oxygen species (ROS) from immunocompetent cells, thus the concentration of neopterin may also be considered as an indirect marker for the amount of immunologically induced oxidative stress (Weiss et al., 1998).

Since neopterin is synthesized by human macrophages, it is of interest to clarify for what purpose these cells have obtained this ability. One of the most important cytotoxic reactions of macrophages stimulated by IFN-gamma is the production of nitric oxide (NO) from L-arginine by the inducible NO synthase (iNOS). iNOS requires 5,6,7,8-tetrahydrobiopterin $\left(\mathrm{BH}_{4}\right)$ as a cofactor. The cleavage of GTP by GTP-cyclohydrolase I results in 
neopterin, yielding 7,8-dihydroneopterin triphosphate, which is a joint precursor of 7,8 dihydroneopterin, neopterin and $\mathrm{BH}_{4}$. Human monocytes/macrophages are the unique source to produce an excess of neopterin derivatives at the rate of $\mathrm{BH}_{4}$ (Hueber et al., 1984). It results in a lower activity of 6-pyrovoyltetrahydropterin synthase, which is an enzyme responsible for the conversion of 7,8-dihydroneopterin triphosphate to $\mathrm{BH}_{4}$. The synthesis of neopterin derivatives instead of biopterin derivatives in humans suggests that neopterin derivatives substitute the regulatory and immunological functions, especially of the $\mathrm{BH}_{4}$-induced $\mathrm{NO}$ generation (Pingle et al., 2008). Thus, because of the excess production of neopterin, $\mathrm{BH} 4$ is available only in a very low concentration in activated human monocytes/macrophages. When $\mathrm{BH}_{4}$ is deficient, NOS shows only oxidase activity: thus active oxygen $\left(\mathrm{O}_{2}^{--}\right)$is generated in greater quantities than NO (Huraux et al., 1999).

Recently, a marked abnormality in pteridine metabolism has been disclosed in a chronic kidney disease (CKD) (Yokoyama et al., 2002), particularly in patients at the most advanced stage of CKD, i.e. the uremic state. Consequently, in uremic patients, the inflammatory process increases because the macrophages and monocytes are stimulated, which, in turn, induces the synthesis of the inducible NOS (iNOS). In those patients, although iNOS synthesis is enhanced when macrophages and monocytes are stimulated, the $\mathrm{BH}_{4}$ level undergoes a relative reduction. Thus, the observed unbalanced iNOS and eNOS concentrations may be closely related to the disorder of pteridine metabolism (Yokoyama et al., 2002).

Since the macrophages are activated in patients with renal failure, one may speculate that the neopterin level is a clinically significant index of both, the renal function and macrophage activity.

Furthermore, increased neopterin has been shown to be predictive of adverse outcomes in patients with myocardial infarction as well as in individuals with chronic stable angina pectoris (Avanzas et al., 2005).

The above-mentioned features of neopterin prompted us to study serum neopterin in patients with all stages of CKD and patients with coronary artery disease. To the best of our knowledge this is the first study that evaluates and compares the serum neopterin concentration in all stages of CKD and among patients suffering from coronary artery disease (CAD) but without kidney dysfunction.

\section{Material and methods}

\section{Study design and participants}

One hundred and twenty five subjects, divided into five groups, were included in the study. The first three groups consisted of patients with different stages of CKD, classified according to The National Kidney Foundation Kidney Disease Outcomes Quality Initiative (NKF $\mathrm{KDOQI}^{\mathrm{TM}}$ ) guidelines (National Kidney Foundation (NKF), 2002) on the basis of their estimated glomerular filtration rate (eGFR), were enrolled.

The first group (CKD1-2) consisted of 25 patients with the initial stages of CKD (eGFR $\geq 90-60 \mathrm{ml} / \mathrm{min} /$ $\left.1.73 \mathrm{~m}^{2}\right)$. In the second group, 25 patients (CKD3-4) with impaired renal function in the middle-level (eGFR $=59-15 \mathrm{ml} / \mathrm{min} / 1.73 \mathrm{~m}^{2}$ ) were included. The last group (CKD5) consisted of 25 patients with end-stage renal disease (ESRD) (eGFR $\left.<15 \mathrm{ml} / \mathrm{min} / 1.73 \mathrm{~m}^{2}\right)$, hemodialized for $36.6 \pm 34.01$ months, 3 times a week for 4.5$5.5 \mathrm{~h} /$ session. After the hemodialysis (HD) session, patients were supplemented with the recombinant human erythopoietin (rHuEPO) and iron. Apart from CKD patients, 25 patients with coronary artery disease (CARD), without any clinical symptoms of renal dysfunction at the time of enrollment to the study or in the past history, were included into the study and formed the fourth group. The fifth group consisted of 25 healthy volunteers (HV) with negative history of an acute or chronic inflammation, and with absence of anemia or any clinical and laboratory symptoms of atherosclerosis. They were characterized by normal renal function, confirmed by no abnormalities in urinalysis, serum level of creatinine in normal ranges and normal eGFR. All the groups were matched for age, gender and race.

Patients with uncontrolled hypertension, diabetes mellitus, active acute infection, immunosuppressive treatment, currently existing malignant tumor or with tumors of over 10 years' history, as well as those currently smoking and/or currently abusing alcohol and those who have used so for over the last 10 years, were excluded from the study.

\section{Laboratory procedures}

Blood samples were drawn from all study participants in the fasting state, between 8:00 and 9:00 a.m. From hemodialyzed patients, blood samples were drawn before the onset of the second hemodialysis session of 
Table 1. Clinical and biochemical characteristics of the studied groups

\begin{tabular}{|c|c|c|c|c|c|c|}
\hline & \multicolumn{4}{|c|}{ Group of patients } & \multirow{3}{*}{$\begin{array}{l}\mathrm{HV} \text { as control } \\
\text { group } \\
(n=25)\end{array}$} & \multirow{3}{*}{$P^{*}$} \\
\hline & \multicolumn{3}{|c|}{$\begin{array}{l}\text { CKD } \\
(n=75)\end{array}$} & \multirow{2}{*}{$\begin{array}{l}\text { CARD } \\
(n=25)\end{array}$} & & \\
\hline & $\begin{array}{l}\text { CKD 1-2 } \\
(n=25)\end{array}$ & $\begin{array}{l}\text { CKD 3-4 } \\
(n=25)\end{array}$ & $\begin{array}{l}\text { CKD5 } \\
(n=25)\end{array}$ & & & \\
\hline Age [years] & $60.1 \pm 8.01$ & $59.9 \pm 8.4$ & $60.1 \pm 10.1$ & $59.4 \pm 9.08$ & $59.5 \pm 11.2$ & NS \\
\hline Males [n ] & 7 & 7 & 7 & 7 & 7 & NS \\
\hline Body mass index: $\left[\mathrm{kg} / \mathrm{m}^{2}\right]$ & $28.19 \pm 3.1$ & $26.3 \pm 2.01$ & $24.7 \pm 3.7$ & $28.3 \pm 3$ & $24.6 \pm 2.1$ & 0.00 \\
\hline $\mathrm{eGFR}\left[\mathrm{ml} / \mathrm{min} / 1.73 \mathrm{~m}^{2}\right]$ & $77.04 \pm 22.9$ & $20 \pm 7.9$ & $5.75 \pm 7$ & $93.22 \pm 20.8$ & $123.6 \pm 17.6$ & 0.00 \\
\hline $\mathrm{HGB}[\mathrm{g} / \mathrm{dl}]$ & $13.31 \pm 3.16$ & $11.76 \pm 1.57$ & $11.41 \pm 1.55$ & $13.83 \pm 1.22$ & $14.02 \pm 0.98$ & 0.04 \\
\hline $\mathrm{RBC}\left[10^{12} / 1\right]$ & $4.65 \pm 0.84$ & $3.92 \pm 0.66$ & $3.69 \pm 0.54$ & $4.42 \pm 0.68$ & $4.71 \pm 0.48$ & 0.00 \\
\hline $\mathrm{HCT}[\%]$ & $43.02 \pm 5.75$ & $35.39 \pm 4.45$ & $32.27 \pm 4.61$ & $40.67 \pm 3.87$ & $44.07 \pm 3.12$ & 0.00 \\
\hline $\mathrm{WBC}\left[10^{9} / 1\right]$ & $7.38 \pm 2.44$ & $7.03 \pm 1.96$ & $6.08 \pm 1.76$ & $6.71 \pm 1.63$ & $5.43 \pm 0.91$ & 0.02 \\
\hline $\operatorname{PLT}\left[10^{9} / 1\right]$ & $205.71 \pm 44.9$ & $227 \pm 81.8$ & $179.35 \pm 72.18$ & $213.32 \pm 41.93$ & $316.73 \pm 73.9$ & 0.00 \\
\hline $\mathrm{Fe}[\mu \mathrm{mol} / 1]$ & $89.2 \pm 43.1$ & $74.26 \pm 27.65$ & $76.33 \pm 28.31$ & $97.91 \pm 42.43$ & $113.45 \pm 23.88$ & 0.00 \\
\hline Ferritin [ng/ml] & $207.6 \pm 111.4$ & $301.74 \pm 229.82$ & $1084.92 \pm 740.57$ & $198 \pm 131.7$ & $209 \pm 110.91$ & 0.00 \\
\hline Total cholesterol [mg/dl] & $217.06 \pm 52.89$ & $182.33 \pm 29.69$ & $179.53 \pm 48.39$ & $191.64 \pm 41.22$ & $188.73 \pm 33.04$ & NS \\
\hline HDL cholesterol [mg/dl] & $56.2 \pm 11.79$ & $58.4 \pm 7.64$ & $46.89 \pm 23.076$ & $43.62 \pm 10.16$ & $70.62 \pm 6.32$ & NS \\
\hline LDL cholesterol [mg/dl] & $168.66 \pm 53.11$ & $120.06 \pm 17.11$ & $105.97 \pm 40.75$ & $118.02 \pm 31.66$ & $93.96 \pm 30.21$ & 0.01 \\
\hline Triglycerides [mg/dl] & $171.15 \pm 70.1$ & $116.66 \pm 24.74$ & $133.35 \pm 50.83$ & $149.96 \pm 75.76$ & $120.72 \pm 37.1$ & 0.02 \\
\hline Glucose [mg/dl] & $93.47 \pm 9.52$ & $91.85 \pm 9.17$ & $86.01 \pm 10.51$ & $97.2 \pm 8.99$ & $79.03 \pm 10.13$ & 0.00 \\
\hline Albumins [g/dl] & $4.0 \pm 0.96$ & $4.13 \pm 1.84$ & $4.33 \pm 0.68$ & $4.33 \pm 0.35$ & $4.24 \pm 0.67$ & NS \\
\hline Total protein $[\mathrm{g} / \mathrm{dl}]$ & $6.6 \pm 1.82$ & $5.5 \pm 1.23$ & $6.63 \pm 0.81$ & $7.39 \pm 0.38$ & $7.21 \pm 0.43$ & $\mathrm{NS}$ \\
\hline Urea [mg/dl] & $30.75 \pm 19.82$ & $93.82 \pm 46.75$ & $121.74 \pm 35.56$ & $38.22 \pm 11.89$ & $27.45 \pm 9.67$ & 0.00 \\
\hline $\begin{array}{l}\text { High sensitivity C-reactive } \\
\text { protein hsCRP [mg/l] }\end{array}$ & $10.75 \pm 8.33$ & $11.0 \pm 12.45$ & $12.32 \pm 18.86$ & $7.06 \pm 2.92$ & $1.18 \pm 1.44$ & 0.00 \\
\hline CA-IMT [mm] & $0.72 \pm 0.16$ & $0.74 \pm 0.11$ & $0.77 \pm 0.32$ & $0.74 \pm 0.19$ & $0.48 \pm 0.20$ & 0.00 \\
\hline
\end{tabular}

${ }^{*}$ CKD1-2 vs. CKD3-4 vs. CKD5 vs. CARD vs. HV assessed by the Kruskal-Wallis test used for nonparametric comparisons; bold font highlights the statistically significant level with $P<0.05$ between the studied groups; NS indicates not significant

the week (and before heparin administration), at the same time as their usual monitoring blood tests were performed.

Blood samples were taken within the same period in fasting patients and in fasting HV. Then the sera samples were immediately separated by centrifugation after clotting and frozen at $-80^{\circ} \mathrm{C}$ before the assay. All samples were stored and processed in an identical fashion.

The study was approved by the Ethical Committee of Poznan University of Medical Sciences, Poland. All study participants were fully informed about the study and were asked to express verbal and written consent prior to the examination. All the experiments were performed in accordance with the Declaration of Helsinki.
Serum neopterin concentration was measured by an enzyme-linked immunosorbent assay (ELISA, BRAHMS, Hennigsdorf/Berlin, Germany) according to the manufacturers' instructions.

All other laboratory assessed parameters were performed using standard methods, according to the manufacture's instruction, at the Central Clinical Hospital Laboratory and in the Laboratory of Kidney Diseases Immunopathology and Tissue Compatibility at Poznan Clinical Hospital No. 2.

Carotid artery intima media thickness (CA-IMT) was measured by The Accuson CV 70 system (Simens, Erlangen, Germany) with a $10 \mathrm{MHz}$ transducer. The distal $1 \mathrm{~cm}$ of the common carotid artery just proximal to the bulb 
was measured by employing a computer analysis system (Medical Imaging Applications, LLC, Coralville, USA).

\section{Statistical analysis}

The results were expressed as means \pm standard deviation (SD). The analyses were performed on the input data, mean values or ranks, depending on the applied tests. The examination of the distribution normality of variables was done using W-Shapiro-Wilk test. Comparisons of the normally distributed variables between the studied groups were performed by unpaired Student $t$-test or for multiple comparisons the one-way analysis of variance (ANOVA) test followed by the post hoc analysis made using the least significance difference (LSD) test. For the multiple comparisons of the variables, not distributed normally, the Kruskal-Wallis test was used. The strength of the relationship between the studied variables was examined using the Pearson's coefficient. Furthermore, a multiple regression analysis was performed to determine independent factors affecting the dependent variable, i.e. the concentration of serum neopterin. All parameters that were significantly associated in a linear manner with serum neopterin were introduced in the model. Moreover, to determine the influencing serum neopterin factors, a multivariable analysis of variance (MANOVA) was performed. $P<0.05$ was considered statistically significant.

All statistical analyses were carried out by the STATISTICA computer software version 8.0 (Stat Soft Inc., Tulsa, OK, USA).

\section{Results}

\section{Characteristics of the studied groups}

The characteristics of the studied groups have been presented in Table 1. There were no differences in age and sex between the participants classified into different groups. Both, the subjects from HV and CKD5 groups, were characterized by a significantly lower body mass index (BMI). Based on the analysis of biochemical data, it can be seen that patients from CKD3-4 and CKD5 groups suffered from anemia and had a lower serum iron level when compared to the other groups. Ferritin concentration in serum was significantly high among patients with $\mathrm{CKD}$, in contrast to CARD and HV having serum ferritin in normal ranges. Moreover, it was found that serum ferritin was increased with the progression of kidney disease. The studied patients differed in lipids parameters. In CKD groups, the serum concentration of LDL cholesterol and triglycerides decreased with the worsening of the renal function, thus hemodialyzed patients showed the lowest serum concentration of these parameters. Similarly, serum glucose was the lowest in the CKD5 group. An inflammatory process confirmed by hsCRP was similar in CKD groups and significantly higher when compared to CARD and HV. Clinical and biochemical characteristics of the studied groups have been shown in Table 1 .

\section{Serum concentration of neopterin and its correlative factors}

The serum concentration of neopterin $[\mathrm{nmol} / \mathrm{l}]$ was significantly increased in CKD patients, particularly in hemodialyzed patients (CKD5 group), and even in patients in the initial stages of CKD was a slightly higher, but not statistically significant than in CARD patients or in HV group, which has been presented in Figure 1.

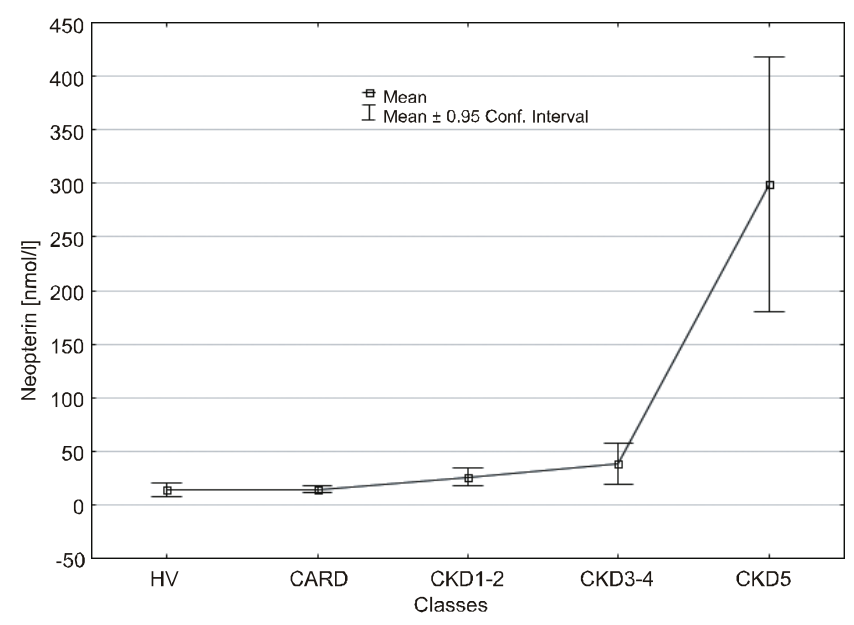

Fig. 1. Differences of the mean values of the serum neopterin concentration and the $95 \%$ confidence interval between all studied classes

In univariate correlative analysis, serum neopterin concentration was positively correlated with BMI, history of arterial hypertension, peripheral systolic blood pressure, HD vintage, CA-IMT, ferritin, urea and hsCRP. Serum IL-18 negatively correlated with $\mathrm{HGB}, \mathrm{RBC}$, serum $\mathrm{Fe}, \mathrm{ALB}$, total protein and eGFR. The selected corre lations, i.e. between neopterin and HD vintage, CA-IMT, eGFR have been presented in Figures 2, 3 and 4, respectively. No correlations between serum neopterin and age, 


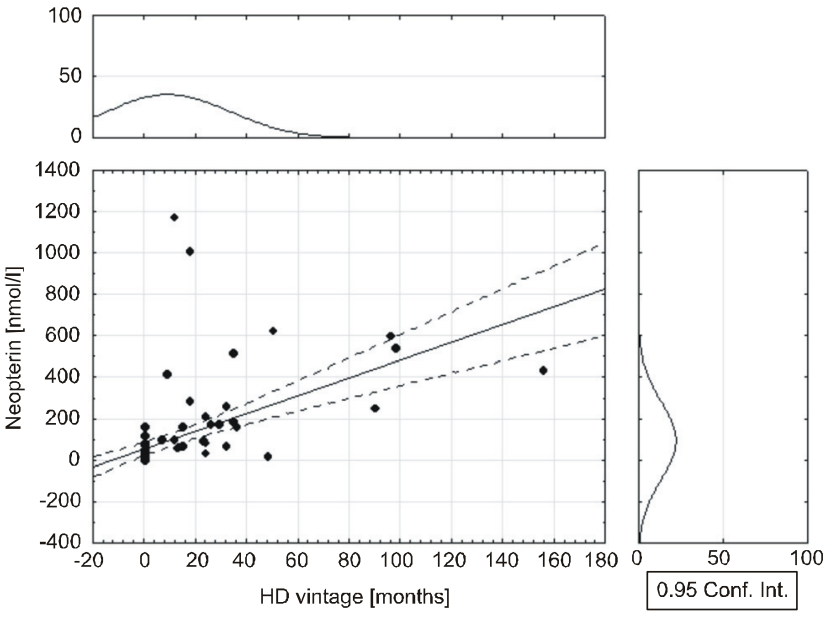

Fig. 2. The scatter diagram (with histograms, i.e. the upper and the right panel which are a graphical way of presenting the empirical distribution characteristics) shows the association between serum neopterin and HD vintage. It illustrates the strong positive correlation $(r=0.54, P=0.00)$ which suggests a strong relationship between these variables. The solid line indicates the linear regression, and the dotted line - the 95\% confidence interval, $r$ - Pearson's correlation coefficient, $P$ - value of the significance level

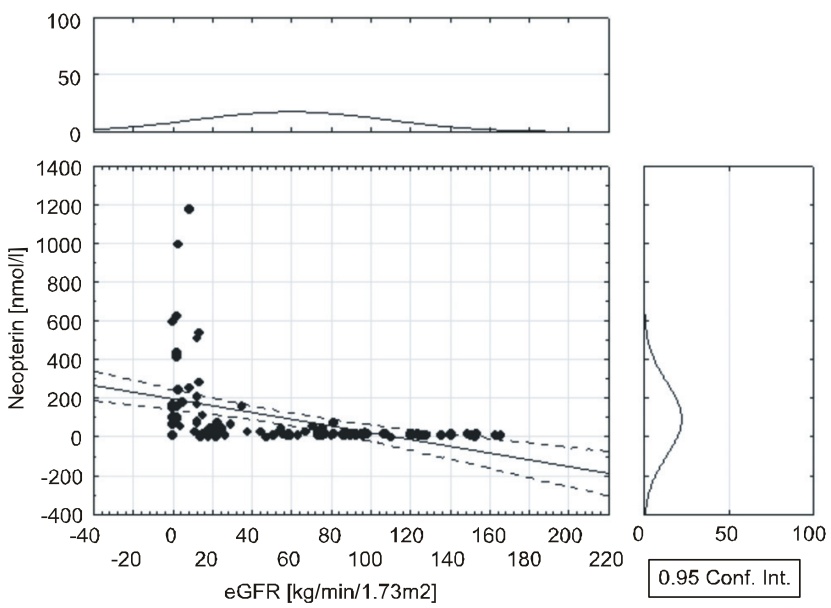

Fig. 3. The scatter diagram (with histograms, i.e. the upper and the right panel which are a graphical way of presenting the empirical distribution characteristics) shows the association between serum neopterin and eGFR. It illustrates the strong negative correlation $(r=-0.44, P=0.00)$ which suggests a strong inverse relationship between these variables. The solid line indicates the linear regression, and the dotted line - the 95\% confidence interval, $r$ - Pearson's correlation coefficient, $P$ - value of the significance level

sex, presence of cardiac disease, history of myocardial infarction/stroke, peripheral diastolic blood pressure, serum glucose and selected lipid metabolism parameters were observed. For detailed information see Table 2.
Table 2. The univariate correlative analysis between serum neopterin concentration and selected parameters, found in all studied groups taken together

\begin{tabular}{l|c|c}
\hline \multicolumn{1}{c|}{ Parameter } & $r$ & $P$ \\
\hline Age & 0.00 & 0.09 \\
\hline Sex & 0.16 & 0.92 \\
\hline Body mass index (BMI) & $\mathbf{0 . 2 1}$ & $\mathbf{0 . 0 2}$ \\
\hline History of arterial hypertension & $\mathbf{0 . 2 4}$ & $\mathbf{0 . 0 1}$ \\
\hline Cardiac disease & 0.45 & 0.63 \\
\hline History of myocardial & -0.08 & 0.36 \\
\hline infarction/stroke & $\mathbf{0 . 3 4}$ & $\mathbf{0 . 0 0}$ \\
\hline Peripheral systolic blood pressure & 0.18 & 0.13 \\
\hline Peripheral diastolic blood pressure & $\mathbf{0 . 5 4}$ & $\mathbf{0 . 0 0}$ \\
\hline HD vintage & $\mathbf{0 . 2 6}$ & $\mathbf{0 . 0 2}$ \\
\hline CA-IMT & 0.04 & 0.69 \\
\hline Glucose & $-\mathbf{0 . 3 9}$ & $\mathbf{0 . 0 0}$ \\
\hline HGB & $-\mathbf{0 . 3 9}$ & $\mathbf{0 . 0 0}$ \\
\hline RBC & $-\mathbf{0 . 3 3}$ & $\mathbf{0 . 0 0}$ \\
\hline Fe & $\mathbf{0 . 4 4}$ & $\mathbf{0 . 0 0}$ \\
\hline Ferritin & -0.07 & 0.50 \\
\hline Triglycerides & -0.08 & 0.45 \\
\hline Total cholesterol & -0.04 & 0.68 \\
\hline HDL cholesterol & -0.08 & 0.41 \\
\hline LDL cholesterol & $\mathbf{- 0 . 3 1}$ & $\mathbf{0 . 0 2}$ \\
\hline ALB & $-\mathbf{0 . 3 5}$ & $\mathbf{0 . 0 0}$ \\
\hline Total protein & $\mathbf{- 0 . 4 4}$ & $\mathbf{0 . 0 0}$ \\
\hline eGFR & $\mathbf{0 . 4 6}$ & $\mathbf{0 . 0 0}$ \\
\hline Urea & $\mathbf{0 . 6 0}$ & $\mathbf{0 . 0 0}$ \\
\hline
\end{tabular}

Bold font highlights statistically significant level with $P<0.05$; $r$ - Pearson's correlation coefficient; $P$-value of the significance level

Moreover, we have found the statistically significant correlations between serum neopterin and selected medications taken by the studied patients, like $\beta$-blockers, acetylsalicylic acid (ASA), Fe (i.v.) and rHuEPO (i.v.). For detailed information see Table 3.

\section{Multivariate regression analysis}

A multivariate regression analysis was applied in order to estimate the impact of individual variables on the serum concentration of neopterin. First, we calculated Pearson's correlation coefficients for serum neopterin concentration and variables likely to have an impact 


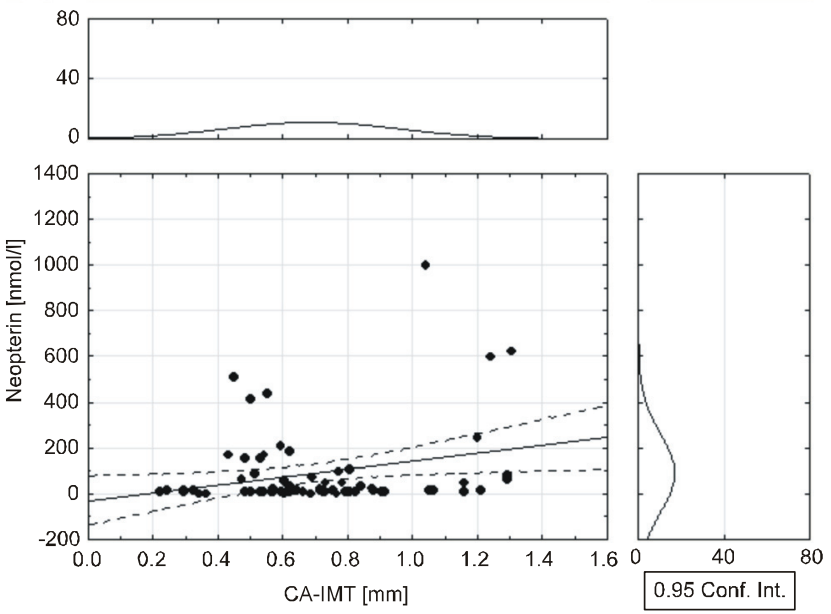

Fig. 4. The scatter diagram (with histograms, i.e. the upper and the right panel which are a graphical way of presenting the empirical distribution characteristics) shows the association between serum neopterin and CA-IMT. It illustrates the strong positive correlation $(r=0.26, P=0.02)$ which suggests a strong relationship between these variables. The solid line indicates the linear regression, and the dotted line - the 95\% confidence interval, $r$ - Pearson's correlation coefficient, $P$ - value of the significance level

on serum neopterin. Then, all the variables that were significantly correlated with serum neopterin concentrations were entered into a stepwise regression models. Those models, after adjustment for urea CA-IMT, peripheral systolic blood pressure, ferritin, hsCRP, HD vintage, BMI, and eGFR proved to be significant and predictors taken together explained $95.89 \%$ of the serum concentration of neopterin $(R$ square $=95.89 \%, F=8.75$, $P<0.05$ and SE of estimate $=65.868)$. Two of the eight predictors included in the model, i.e. serum urea and serum ferritin, showed significant effects $(P=0.018$ and $P=0.006$, respectively). The results of the final step of this analysis are presented in Table 4 .

\section{Multivariable ANOVA (MANOVA) analysis}

In this analysis, it was discovered that three parameters, i.e. CA-IMT, hsCRP and eGFR, taken together, significantly influenced the serum neopterin concentration $(P=0.00, F=2226.624$, with a standard error of 119.027) (data not shown).

\section{Discussion}

Patients suffering form CKD, especially those who are hemodialyzed, demonstrate severe dysregulation in the immune system combining an immunodeficiency
Table 3. The univariate correlative analysis between serum neopterin concentration and selected medications taken by the studied patients revealed in all studied groups taken together

\begin{tabular}{l|c|c}
\hline & $r$ & $P$ \\
\hline Lipid-lowering drugs, i.e. statins & -0.080 & 0.389 \\
\hline $\begin{array}{l}\text { Angiotensin-converting enzyme } \\
\text { inhibitors (ACEI) }\end{array}$ & -0.037 & 0.694 \\
\hline$\beta$-blockers & $\mathbf{0 . 2 6 0}$ & $\mathbf{0 . 0 0 6}$ \\
\hline Acetylsalicylic acid (ASA) & $-\mathbf{0 . 1 8 7}$ & $\mathbf{0 . 0 4 8}$ \\
\hline Nitrates & -0.132 & 0.167 \\
\hline Fe (i.v.) & $\mathbf{0 . 4 3 2}$ & $\mathbf{0 . 0 0 0}$ \\
\hline rHuEPO (i.v.) & $\mathbf{0 . 2 5 1}$ & $\mathbf{0 . 0 0 8}$ \\
\hline
\end{tabular}

Bold font highlights a statistically significant level with $P<0.05$; $r$-Pearson's correlation coefficient; $P$ - value of the significance level

state and activation of $\mathrm{T}$ and $\mathrm{B}$ cells, and monocytes (Friedlander et al., 1996). These immune disturbances progress in parallel with the degree of renal dysfunction, and are amplified in patients with the most advanced stage of CKD, i.e. in ESRD. In our study it was found that neopterin, which gives us an insight into the disturbances in the cellular immune system, was fifteen times higher in patients in the most advanced stages of kidneys dysfunction in comparison to patients in the earlier stages of $\mathrm{CKD}$ and patients with coronary artery disease without $\mathrm{CKD}$. Interestingly, in early stages of CKD (CKD1-2, CKD3 groups) and among CAD patients without $\mathrm{CKD}$, the serum neopterin concentrations were similar to those obtained from healthy volunteers.

The results of neopterin measurements are in agreement with the current state of knowledge. They emphasize the important role that cellular immune system plays in CKD. The increased level of serum neopterin in ESRD can be explained by the impaired excretion as well as increased production of the compound due to systemic inflammatory processes. Our results have verified the knowledge received from other studies in which both serum and urinary neopterin levels were used as a diagnostic or as a prognostic marker in several pathologies including renal diseases (Berdowska, and Zwirska-Korczala, 2001, Lhee at al., 2006, Asci et al., 2010).

Apart from confirming the existence of high levels of neopterin in hemodialyzed patients, we have also found that treatments with rHuEPO, iron, $\beta$-blockers and acetylsalicylic acid (ASA) affect serum concentrations of 
Table 4. Regression coefficients and standard errors of the multiple linear regression with changes in the serum concentration of neopterin as dependent variables and urea, CA-IMT, peripheral systolic blood pressure, ferritin, hsCRP, HD vintage, BMI and eGFR as independent variables $R=97.9 \%, R$ square $=95.89 \%$, standard error of the estimate $65.868, P<0.05$. This model predicts neopterin serum concentration with multiple $R$ square $95.89 \%$

\begin{tabular}{|c|c|c|c|c|c|c|}
\hline & \multicolumn{2}{|c|}{ Standardized coefficient } & \multicolumn{2}{|c|}{ Unstandarized coefficient } & \multirow[b]{2}{*}{$t$} & \multirow{2}{*}{$\begin{array}{c}\text { Significance level } \\
\text { ( } P \text {-value })\end{array}$} \\
\hline & beta & $\begin{array}{c}\text { standard beta } \\
\text { error }\end{array}$ & B & $\begin{array}{l}\text { standard B } \\
\text { error }\end{array}$ & & \\
\hline Constant & & & -1554.54 & 485.32 & -3.21 & 0.05 \\
\hline Urea & 1.51 & 0.32 & 8.31 & 1.77 & 4.68 & 0.01 \\
\hline CA-IMT & -0.07 & 0.34 & -34.71 & 174.57 & -0.19 & 0.85 \\
\hline Peripheral systolic blood pressure & 0.20 & 0.13 & 1.57 & 1.06 & 1.47 & 0.23 \\
\hline Ferritin & -0.78 & 0.27 & -0.23 & 0.08 & -2.89 & 0.006 \\
\hline hsCRP & -0.33 & 0.17 & -10.29 & 5.35 & -1.92 & 0.15 \\
\hline HD vintage & 0.66 & 0.34 & 5.19 & 2.66 & 1.94 & 0.14 \\
\hline BMI & 0.49 & 0.27 & 27.02 & 14.84 & 1.82 & 0.16 \\
\hline eGFR & 0.28 & 0.25 & 5.64 & 4.88 & 1.15 & 0.33 \\
\hline
\end{tabular}

Bold font highlights a statistically significant level with $P<0.05$

neopterin. Results of research into this phenomenon are conflicting (for comparison, refer to Asci et al., 2010). There is a growing interest towards the non-erythropoietic, tissue-protective and restorative actions of erythropoietin (EPO) (Ghezzi and Mengozzi, 2007). The accumulated data reveal that EPO exerts pleiotropic activities and suggests a potential novel use of rHuEpo as an immunomodulator (Godai et al., 1991). Further studies are needed to show the role of EPO in the immune system, particularly in the cell-mediated immunity. In contrast to the results obtained by Walter et al. (2003) and Neurauter et al. (2003), in the present study, we have found that treatments of statins did not affect the neopterin levels. In the above-mentioned studies, statins (3-Hydroxy-3-methylglutaryl-coenzyme A (HMG-CoA) reductase inhibitors), having anti-inflammatory properties, were shown to lower the neopterin production in a casecontrol study of patients undergoing coronary angiography. This difference may be due to their treatment with different types of statins and use of higher dosages of drugs than in our study.

Only limited information has been available so far with regard to the utility of neopterin as a diagnostic and prognostic marker in cardiovascular diseases. It has been postulated that cellular immunity might be activated and thus neopterin concentrations might be increased in patients with congestive heart failure (Grammer et al., 2009). In our study, we have found that hemodialyzed patients suffering from congestive heart failure reveal higher neopterin serum concentrations in comparison to other hemodialyzed patients (data not shown). Moreover, we did not observe any differences in serum neopterin levels between living patients and those who died within 12 months (data not shown). Interestingly, neopterin serum concentrations in all hemodialyzed patients were much higher than in patients suffering from coronary artery disease, even those with congestive heart failure. This highlights the fact that immunological disturbances under dialysis treatment are crucial for the changes in serum neopterin levels. On the other hand, even if we have not found any statistically signifiant associations between serum neopterin and triglycerides, and serum neopterin and cholesterol and its fractions, the existence of an influence of neopterin on atherosclerosis, characterized by the well-established immunological disturbances seems obvious. A positive correlation between serum neopterin and CA-IMT confirms the above findings. Increased serum neopterin levels were also observed by other researchers in patients with carotid atherosclerosis and peripheral artery disease (Tatzber et al., 1991).

Furthermore, we have found that in hemodialyzed patients, serum neopterin concentrations were correlated positively with the length of time of the dialysis (HD 
vintage). During dialysis, the immunulogiacal disturbances increase, so the relationship between HD vintage and serum neopterin seems to be quite obvious. However, the results obtained form other studies are inconclusive (see Godai et al., 1991, Asci et al., 2010).

Other associations found in our study i.e. the one between serum neopterin and eGFR suggest that in patients with CKD and CAD, the neopterin levels in serum are closely linked with glomerular filtration. Moreover, one can see the impact of anemia and iron disturbances on the serum neopterin concentration in the studied patients. It is known that iron metabolism disturbances are closely associated with the presence of an oxidative stress (Formanowicz, 2011), and neopterin itself could contribute to the changes in an oxidative stress as its main function is the modulation of this process.

The results obtained from our study also emphasize the importance of the influence of an inflammatory process on serum neopterin (see correlation between serum neopterin and hsCRP). On the other hand, model that best described the variability of the neopterin comprised the predictors that were associated with inflammation as well as a kidney disease. Among them, only urea and ferritin (acting as a positive acute phase protein) showed a significant influence. Moreover, it was found in a multivariate analysis that CA-IMT, hsCRP and eGFR taken together significantly influenced serum neopterin concentration.

So it seems that although many clinical and laboratory factors may influence serum neopterin, the crucial ones are those which derive from the impaired renal function and immunological and inflammatory disturbances coming from hemodialysis. In this study, we observed that the more impaired the renal function, the higher increase in serum neopterin concentration.

\section{References}

Asci A., Baydar T., Catinkaya R., Dolgun A., Sahin G. (2010) Evaluation of neopterin levels in patients undergoing hemodialysis. Hemodial. Int. 14: 240-246.

Avanzas P., Arroyo-Espliguero R., Quiles J, Roy D., Kaski J.C. (2005) Elevated serum neopterin predicts future adverse cardiac events in patients with chronic stable angina pectoris. Eur. Heart J. 26: 457-463.

Berdowska A., Zwirska-Korczala K. (2001) Neopterin measurement in clinical diagnosis. J. Clin. Pharm. Ther. 26: 319329 .
Formanowicz D. (2011) Do changes in iron metabolism contribute to the acceleration of the atherosclerosis process? Biotechnologia, 92: 180-192.

Friedlander M.A., Witko-Sarsat V., Nguyen A.T., Wu Y.C., Labrunte M., Verger C., Jungers P., Descamps-Latscha B. (1996) The advanced glycation endproduct pentosidine and monocyte activation in uremia. Clin. Nephrol. 1996; 45: 379-382.

Fuchs D., Weiss G., Reibnegger G., Wachter H. (1992) The role of neopterin as a monitor of immune activation in transplantation, inflammatory, infectious, and malignant diseases. Crit. Rev. Clin. Lab. Sci. 29: 307-341.

Fuchs D., Weiss G., Wachter H. (1993) Neopterin, biochemistry and clinical use as a marker for cellular immune reactions. Int. Arch. Allergy Immunol. 101: 1-6.

Ghezzi P., Mengozzi M. (2007) Activities of erythropoietin on tumors: an immunological perspective. Eur. J. Immunol. 37: 1427-1430.

Godai K., Uemasu J., Kawasaki H. (1991) Clinical significance of serum and urinary neopterins in patients with chronic renal disease. Clin. Nephrol. 36: 141-146.

Grammer T.B., Fuchs D., Boehm B.O., Winkelmann B.R., Maerz W. (2009) Neopterin as a predictor of total and cardiovascular mortality in individuals undergoing angiography in the Ludwigshafen risk and cardiovascular health study. Clin. Chem. 55: 1135-1146.

Hueber C., Batchelor J.R., Fuchs D., Hausen A., Lang A., Niederwieser D., Reibnegger G., Swetly P., Troppmair J., Wachter H. (1984) Immune response-associated production of neopterin. Release from macrophages primarily under control of interferon gamma. J. Exp. Med. 160, 310-316.

Huraux C., Makita T., Kurz S., Yamaguchi K., Szlam F., Tarpey M.M., Wilcox J.N., Harrison D.G., Levy J.H. (1999) Superoxide production, risk factors, and endothelium-dependent relaxations in human internal mammary arteries. Circulation 99: 53-59.

Lhee H.Y., Kim, H., Joo, K.J., Jung, S.S, Lee, K.B. (2006) The clinical significance of serum and urinary neopterin levels in several renal diseases. J. Korean Med. Sci. 21: 678-682.

Margreiter R., Fuchs D., Hausen A., Huber C., Reibnegger G., Spielberg M., Wachter H. (1983) Neopterin as a new biochemical marker for diagnosis of allograft rejection. Experience based upon evaluation of 100 consecutive cases. Transplantation. 36: 650-653.

Murr C., Widner B., Wirleitner B., Fuchs D. (2002) Neopterin as a marker for immune system activation. Curr. Drug. Metabol. 3: 175-187.

National Kidney Foundation (NKF) Kidney Disease Outcome Quality Initiative (K/DOQI), Advisory board: K/DOQI clinical practice guidelines for chronic kidney disease: evaluation, classification, and stratification. Kidney Disease Outcome Quality Initiative. Am. J. Kidney. Dis. 2002, 39: $1-246$.

Neurauter G., Wirleitner B., Laich A., Schennach H., Weiss G., Fuchs D. (2003) Atorvastatin suppresses interferon-gamma- 
induced neopterin formation and tryptophan degradation in human peripheral blood mononuclear cells and in monocytic cell lines. Clin. Exp. Immunol. 131: 264-267.

Pingle S.K., Tumane R. G., Jawade A.A (2008) Neopterin: Biomarker of cell-mediated immunity and potent usage as biomarker in silicosis and other occupational diseases. Indian J. Occup. Environ. Med. 12: 107-111.

Singh K-K.B., Wan-Nurfahizul-Izzati W., Ismail A. (2010) Serum neopterin is elevated in patients infected with Shigella. Gut Pathogens 2: 9.

Tatzber F., Rabl H., Koriska K., Erhart U., Puhl H., Waeg G., Krebs A., Esterbauer H. (1991) Elevated serum neopterin levels in atherosclerosis. Atherosclerosis 89: 203-208.
Walter R.B, Fuchs D., Weiss G., Walter T.R., Reinhart W.H. (2003) HMG-CoA reductase inhibitors are associated with decreased serum neopterin levels in stable coronary artery disease. Clin. Chem. Lab. Med. 41: 1314-1319.

Weiss G, Widner B, Zoller H, Schobersberger W, Fuchs D. (1998) Immune response and iron metabolism. Br. J. Anaesth. 81: 6-9.

Yokoyama K., Tajima M., Yoshida H., Nakayama M., Tokutome G., Sakagami H., Hosoya T. (2002) Plasma pteridine concentrations in patients with chronic renal failure. Nephrol. Dial. Transplant. 17: 1032-1036. 